\title{
Zur Frage der Excision der syphilitischen Initialsklerose.
}

\author{
Von \\ Prof. Heinrich Auspitz
}

in Wien.

Der im Jahre 1877 in dieser Vierteljahresschrift von mir veröffentlichten Abhandlung über diesen Gegenstand hat sich eine Reihe von Arbeiten und Experimenten anderer Forscher über dasselbe Thema angeschlossen. Einige derselben sind in dieser Vierteljahrsschrift 1879, 3. Heft, pag. 424, und 4. Heft, pag. 637 u. ff. im Auszuge mitgetheilt worden ${ }^{1}$ ). In dem weiter unten folgenden Berichte wird auch über andere, mir seither zur Kenntniss gekommene Experimente von Rydygier und Klink aus dem Jahre 1879, sowie über jene von Chadzynski (veröffentlicht im 3. Hefte der Annales de Dermatologie, Juli 1880) und endlich über jene von Zeissl (Wiener med. Presse Nr. 27, 28,29, 1880) referirt werden.

Für die Lehre vom syphilitischen Contagium mussten sich sowohl aus den erwähnten Excisionen, als auch aus den anatomischen Befunden, welche ich in Gemeinschaft mit Unna erhalten und dargestellt habe ${ }^{2}$ ), gewisse Folgerungen von weittragender Bedeutung ergeben. Dieselben sind von mir in einem Aufsatze in

') Es sind dies die Excisionen, welche Pospelow, Th. Kölliker, Unna im J. 1878, Folinea im J. 1879 vorgenommen haben.

$\left.{ }^{2}\right)$ Diese Vierteljahrsschrift 1877, 1. Heft: Ueber die An atomie der syphilitischen Initialsklerose ron H. A uspitz in Wien und P. Unna in Hamburg. - Ferner von Unna allein 1878, ebenda: Ein weiterer Beitrag zur Anatomie der syphilitischen Initialsklerose (4. Heft). 
der Wiener medic. Presse (excerpirt in dieser Vierteljahrsschrift 1879 , 2. und 3. Heft, pag. 434 u. ff.) gezogen worden.

Ich habe vorläufig nicht die Absicht, auf das Meritorische weder meiner Excisionen, noch der von Unna und mir studirten anatomischen Verhältnisse, noch auf die sich daran knüpfenden nosologischen Corollarien abermals des Näheren einzugehen. Dagegen erscheint es mir theils aus sachlichen, theils aus persönlichen Gründen geboten, den zwei jüngsten Publicationen über das in Rede stehende Thema von Chadzynski und Zeissl einige Bemerkungen zu widmen.

Herr Dr. Chad zynski hat vom October 1877 bis Jänner 188030 Excisionen von Initialsklerosen vorgenommen. Seine Resultate waren: 7 vollständige Erfolge, 16 vollständige Misserfolge, 6 Fälle, die sich der Beobachtung entzogen, 1 noch unentschiedener. Also 7 (von 23) Erfolge, d. i. etwas über 30\%.

Man sieht, dass das Resultat als ein für die Excision günstiges angesehen werden kann und der Autor unterlässt es auch nicht, die entsprechenden Schlüsse daraus zu ziehen, welche sich im Grossen und Ganzen an die von mir seiner Zeit ausgesprochenen anschliessen, abgesehen freilich von einigen Punkten, für welche ich die Verantwortung keineswegs auf mich nehmen möchte.

Was ich aber hier hervorheben will, betrifft etwas Anderes. Es ist die oberflächliche Art und Weise, mit welcher der Autor die Literatur des Gegenstandes, dem er doch so viel Zeit gewidmet hat, behandelt, oder besser dem französischen Leserkreise gegenüber misshandelt. Wenn Herr Chadzynski als „ancien interne des hôpitaux de Lyon, médecin de l'hôpital général de Lemberg (Gallicie autrichienne)" - so unterzeichnet er seinen Aufsatz in den "Annales de Dermatologie" - seine wissenschaftlichen Arbeiten in französischer Sprache publiciren will, so wird gewiss Niemand dagegen eine Einwendung erheben. Aber so viel dürfen die deutschen und österreichischen Autoren, welche er eitirt und deren Publicationen er seinen Arbeiten zu Grunde legt, wohl erwarten, dass er sie im deutschen Original gelesen habe, oder wenn ihm die deutsche Sprache ganz unbekannt sein sollte, dass er in der österreichischen Stadt Lemberg irgend ein Individuum finde, welches ihm derlei deutsche Aufsätze zu übersetzen im Stande wäre. Herr Chadzynski würde es dadurch vermieden haben, die in meiner 
Arbeit enthaltene Literatur in arger Weise zu entstellen. Ich bin gar nicht im Stande auf die Details einzugehen; als Muster will ich nur hervorheben, dass eine Publication v. Sigmund's aus der Wiener med. Wochenschrift vom J.1867, deren Quelle ich genau citirt, Herr Chadzynski aber falsch reproducirt hat, (Wien. med. Presse) in einer Weise dargestellt ist, als hätte v. Sigmund 39 "Zerstörungen von Initialsklerosen" gemacht, darunter 35 mit günstigem Erfolge! Die bezügliche Arbeit v. Sigmund's hat aber gar nichts mit Initialsklerosen zu thun, sondern nur mit insonten Excoriationen, welche angeblich mit syphilitischer Materie in Berührung gekommen und gleich oder bald nachher von Sigmund geätat worden waren. Ueberdies sind auch noch die Zahlen vollkommen falsch wiedergegeben. Und nun führt Herr Dr. Chadzynski diese Ziffern in einer von ihm construirten Tabelle der Excisionserfolge auf und bringt mit Hilfe noch anderer Unrichtigkeiten schliesslich heraus, dass in der Literatur von 141 ,ihm bisher bekannten" Excisionen 77 Erfolg gehabt, hätten. Auf diese Weise ist in der That mein Percentverhältniss (14 von 28) glücklich erreicht - freilich auf Kosten der Walrheit. Ich will nicht weiter darauf eingehen, dass Vf. auch mit meiner Priorität die sonderbarsten Kunststücke macht: dass er meinem Freunde Unna Sätze aus meiner Abhandlung über die Excision zuschreibt: dass er unsere gemeinschaftliche Arbeit über die Anatomie der Sklerose mit Hartnäckigkeit Unna allein und Theile daraus ihm und Cornil in Paris (dessen Leçons sur la Syphilis 1879 erschienen sind) oder auch dem letzteren allein zuschiebt aber darauf will ich aufmerksam machen, dass die ganze Darstellung des Gegenstandes sammt den daran geknüpften Reflexionen des Autors nur ein vollständig unrichtiges Bild der ganzen Frage $\mathrm{zu}$ geben geeignet ist.

Mag Herr Dr. Chadzyn ski sich immerhin durch seine etwaige Unkenntniss der deutschen Sprache und die daraus hervorgehende Nothwendigkeit die Quellen aus fremder Darstellung ("A uspitz cité par Klink" heisst es einmal im Aufsatze) zu citiren entschuldigt halten - für mich geht darans die Nothwendigkeit hervor, auf diese Thatsache aufmerksam za machen, damit nicht die Kenntniss des Standes der wichtigen Fragen, um die es sich handelt, dem ärztlichen Publikum in Frankreich und daher 
auch in vielen anderen Ländern durch ein so getrübtes Medium vermittelt werde.

Bemerkungen anderer Art sind es, wolche ich an eine Arbeit üher denselben Gegenstand zu knüpfen habe, welche mein geehrter College, Prof. Zeissl in Wien, im Jabresbericht des allgemeinen Krankenhauses und überdies noch separat in der Wiener medic. Presse vor Kurzem veröffentlicht hat.

Hier handelt es sich, wie der Anszug in unserem "Berichte" zeigt, um etwas das Wesen Betreffendes: um Gegenexperimente, welche der Autor den meinigen und anderen gegenüber angestellt hat und um die Schlüsse, oder richtiger gesagt, eine ganze Theorie der Syphiliscontagion, welche der Autor daran knüpft.

Was zunächst die Experimente betrifft, ist die Zahl der von Zeissl beschriebenen nur fünf - da ihm von den zahlreichen Fällen mit nachfolgender Syphilis, die er früher zu beobachten Gelegenheit hatte, nach seinen Angaben keine Krankengeschichte zu Gebote stebe. Ich glaube sicherlich nicht zu irren, wenn ich diese "Excisionen" für Circumcisionen bei phimotischen, gangränescirenden u. dgl. Schankern halte, deren Excision ausschliesslich zum Heilzwecke, natürlich unter ganz anderen Verhältnissen und unter ganz anderem Gesichtswinkel der Beobachtung gemacht wurde, als dies für die präcise Beantwortung eines naturwissenschaftlichen Themas nothwendig wäre. Ich meine aus diesem Grunde, dass der Autor Recht gethan hat, diese Fälle, selbst wenn ihre Krankengeschichten ihm zu Gebote stünden, der wissenschaftlichen Verwerthung zu entzieben. Ebenso ist es nux correct, wenn er weitere vier Fälle, welche nicht von ihm, sondern von befremndeten Collegen operirt und beobachtet wurden, gleichfalls ohne Berücksichtigung lässt und sich somit auf seine eigenen fünf Fälle beschränkt.

Es sei mir aber nunmehr gestattet, mit wenigen Worten die Hauptmomente dieser Fälle zu reproduciren:

1. Fall: Excoriation, seit dem 10.Februar 1874 zuerst bemerkt, die Sklerose excidirt (wann ist nicht angegeben). Nach 3 Monaten, die Patient grösstentheils in Paris zubrachte, ein Erythema papulosum ohne vorausgegangene Verhärtung der Wundfläche.

2. Fall: Eine Sklerose mit Leistendrüsenschwellung (seit wann 
angeblich bestehend?), am Tage nach der Aufnahme (27. April 1880) excidirt - keine Verhärtung der Wundfläche. Am 6. Mai, d. i. also 6 Tage nach der Operation, war ein maculo-papulöses Syphilid entwickelt.

3. Fall: Ein sklerosirtes Präputialgeschwür, angeblich seit 9. März 1880 bestehend mit beiderseitigen Leistendrüsenschwellungen, wurde am 11. April excidirt. Am 18. April, also 7 Tage nach der Excision, bei noch nicht vernarbter Wunde Roseola an beiden Brustseiten.

4. Fall: Auf der Haut des circumcidirten Gliedes eines am 5. December 1879 aufgenommenen Kranken eine angeblich seit 4 Wochen bestehende Sklerose, welche am 6 . December excidirt wurde. Heilung per primam intentionem. Am 18. December, also 12 Tage nach der Operation ein papulöses Syphilid.

5. Fall: Eine seit 7 Wochen bestehende, von Bubonen begleitete Sklerose auf der Gliedhaut wurde am 19. Mai 1880 exeidirt, ohne nachfolgende Verhärtung der Narbe. Am 25. Juni fanden sich auf dem Scrotum, an beiden Wangen und der Zunge nässende Papeln. ( $0 \mathrm{~b}$ inzwischen Roseola des Stammes, Angina faucium u. dgl. aufgetreten war, ist nicht angegeben.)

Es wird für die Kritik des Werthes dieser fünf Fälle ausreichen, wenn ich Folgendes bemerke: a) dass der erste im höchsten Grade ungenau geschildert ist (es fehlt ja sogar das Datum der Excision!); b) dass im 2., 3. und 4. Falle die Erscheinungen allgemeiner Infection schon 6,7 und 12 Tage nach der Operation aufgetreten sind, dass also offenbar die Excision zu einer Zeit gemacht wurde, wo die Allgemein-Infection schon ein Fait accompli gewesen sein oder wenigstens die Wahrscheinlichkeit, sie durch die Excision zu verhüten, nahezu $=0$ sein musste; c) dass also höchstens der letzte Fall zur Verwendung geeignet sein könnte, obgleich auch hier die Krankengeschichte im hohen Grade lückenhaft erscheint. Dabei fällt es mir gar nicht ein, die Diagnose der Sklerose in einem dieser Fälle, selbst im ersten nicht, in Zweifel ziehen zu wollen, wozu - einem so namhaften Syphilidologen gegenüber - keine Berechtigung vorliegt.

Aber die Frage drängt sich doch sicherlich jedem nnparteïschen Prüfer auf, wie der Autor es verantworten kann, auf solche Fälle hin die positiven Erfolge Anderer anzuzweifeln; die Frage als "für 
seine Person abgeschlossen" zu erklären und das Axiom: die Initialsklerose sei stets der Ausdruck der schon vollzogenen Durchseushung - als unangetastet zu proclamiren? Ich bedaure sagen zu müssen, dass die hier von dem geehrten Collegen befolgte wissenschaftliche Methode den fundamentalsten Forderungen, welche man an das Experiment stellen muss, nicht entspricht, abgesehen davon, dass auch die klinische Beschreibung und die kritische Belenchtung der Vorarbeiten viel zu wünschen übrig lässt. ${ }^{1}$ )

Wenn ich nunmehr den theoretischen Theil des Zeissl'schen Aufsatzes in Betracht ziehe, so führt der Autor zuerst zwei „Beweise mehr historischer Art gegen die locale Natur der Sklerose an. Der erste, dass Renault 184813 Pferden Rotz eingeimpft, darauf die Impfstelle mit dem Glüheisen zerstört habe und dass doch alle 13 Pferde Rotz bekamen.

Dieses Experiment mag nun für den Rotz stichhaltig sein, a. h. es mag wahrscheinlich machen, dass die Incubation des Rotzgiftes eine sehr kurze ist - wie aber daraus zu folgern sei, dass auch das Syphilisgift schon einige Stunden nach der Uebertragung in den Stoffwechsel übergegangen sein müsse, ist nicht recht einzuseben. Weil die Erde 365 Tage braucht, um den Kreislauf um die Sonne zu vollenden, wird darum Jemand behaupten wollen, dass der Uranus für diese kosmische Aufgabe keine längere Zeit brauchen dürfe? Und andererseits handelt es sich hier keineswegs um den Satz, ob etwa die Cauterisirung der Imp fstelle innerhalb 24 Stunden nach der Syphilis-Einimpfung den Uebergang des Giftes in den Stoffwechsel verhindere. Aber nur eine solche Fragestellung würde jener für den Rotz aufgestellten analog sein, nicht aber die Frage von der Excision der syphil.

1) Der Autor gibt z. B. an, dass meine 33 Excisionsfalle "nach 3 Monatewährender Beobachtung günstige Resultate ergaben, wälrend doch die 14 von mir als Erfolge bezeichneten Fälle 14, 11, 12, 9, 16, 11, $15,8,4,6,4,9,4,4 \frac{1}{2}$ Monate in Beobachtung gestanden waren, also kein einziger drei Monate! Eine in einer späteren Nummer der "med. Presse" auf meine directe Reclamation hin erfolgte Berichtigung kann wohl die Loyalität des Autors nach dem Druck seines Anfsatzes, nicht aber die wünschenswerthe Genanigkeit bei dessen Abfassung constatiren. 
Sklerose, welche der verehrte Herr College auf so einfache Weise beantwortet zu haben der Meinung ist.

Der zweite "directe Beweis", den Zeissl anführt, ist eine im Jahre 1861 vorgekommene Impfung mit Syphiliseiter auf den Oberschenkel eines Gesunden, bei welchem nach genau drei Wochen Impfpapeln auitraten, nach weiteren 11 Tagen die betreffenden Leistendrüsen empfindlich und vergrössert wurden, und vor der Drüsenschwellung Probeimpfungen mit "syphilitischem Secrete" vorgenommen worden waren, die nicht hafteten - zum Beweise, "dass der Organismus zur Zeit des Auftretens des syphilitischen Primäreffectes mit Syphilis durchseucht war, also nicht gleichzeitig ein zweites Mal Syphilisgift aufnehmen konnte."

Auch dieser, schon in des Verfassers Lehrbuche citirte Fall ist gänzlich obne Beweiskraft für den Satz, dass die Induration das erste Symptom der schon vollzogenen Durchseuchung sei. Zuerst muss ich darauf hinweisen, dass der positive oder negative Erfolg von Probeimpfungen mit "syphilitischem Secrete" (überdies ohne nähere Charakterisirung desselben) auf einen Syphilitischen ganz und gar Nichts zu beweisen geeignet wäre, weil ja bekanntlich die seither stattgefundenen zahlreichen Experimente (zuerst jene von Bidenkap, dann von vielen Anderen) von SyphiliseiterUeberimpfung auf Syphilitische gezeigt haben: a) dass dieselbe in der Regel sofort in Generationen wieder inoculable Geschwüre (Chancroide, weiche Schanker?); b) in Ausnahmsfällen aber auch dieselbe Wirkung wie bei Gesunden hervorrufen, d. i. nach einer gewissen Zeit Papeln und dann selbst indurirte Schanker hervorbringen kann.

Ueberdies aber ist zu constatiren, dass Syphilis-Ueberimpfungen sowohl bei Gesunden als bei Syphilitischen bisweilen ganz wirkungslos bleiben.

Das Letztere erscheint selbstverständlich, wenn man bedenkt, wie viel Mühe und Zeit es kostete, bis die Thatsache von der Möglichkeit der Uebertragung secundärer Syphilis. Symptome auf Gesunde anerkannt wurde, und ferner, dass die Möglichkeit dieser Ansteckung gewisser speciell für sie günstiger Momente bedarf, welche in den meisten Fällen nicht vorhanden sind. Anders wäre es kaum begreiflich, warum nicht das ganze Mensehengeschlecht heute syphilitisch sei. In der That hat sich zu einer Zeit, als diese 
Uebertragungs-Bedingungen günstigere (die Acuität stärker) waren, d. i. zur Zeit des Auftretens der Seuche in Europa am Ende des 15. Jahrhunderts, die Syphilis ganz so, wie heute die acuten Exantheme u. s. w. in Form von Endemien und Epidemien fortgepflanzt. Wenn dies demnach für die Uebertragung nicht blos auf Syphilitische, sondern auch auf Gesunde gilt, so ist daraus zu schliessen, dass das von Zeissl angeführte Impfexperiment keine Beweiskraft gerade für denjenigen Punkt hat, anf welchen es abzielt, nämlich auf die These von der schon vorhanden gewesenen Durchsenchung des Organismus im angeführten Falle Zeissl's.

Der "directe Beweis" des Autors ist aber nicht in diesem Punkte allein angreifbar, sondern es sind noch einige andere gewichtige Einwendungen gegen ihn zu erheben. Fr hat drei Wochen nach der Impfung "Impfpapeln" constatirt, eine nähere Beschreibung des Aussehens und Verlaufes derselben ist nicht gegeben. Aber soviel ist sicher, dass sie keine "Initialsklerosen" waren. Nun ist aber meines Wissens von nichts Anderem als von Excision der Sklerose die Rede gewesen und die Schlüsse, welche ich seiner Zeit aus meinen Resultaten gezogen habe und welche Zeissl bekämpft, haben sich ausschliesslich auf die Initialsklerose bezogen. Ich habe nie behauptet, dass die Papel, ob sie nun auf der Impfstelle oder anderswo auftrete, oder dass die Plaques und Condylome u. s. w., welche ja ebenfalls Papeln sind, nicht der Ausdruck der syphilitischen Diathese seien; ja ich habe schon in meiner Contagienlehre, welche im Jahre 1866 ersehienen ist, ausdrücklich auf den wesentlichen Unterschied zwischen beiden hingewiesen ${ }^{1}$ ) und aus den Experimenten wie aus den klinischen Erfahrungen den Nachweis geführt, „dass zwischen der Impfipapel und der Induration als solche in dem Sinne, wie sie von den Classikern der dualistischen Lehre definirt wird, keine Gemeinschaft herrscht." In der That haben auch die neueren anatomischen Untersuchungen von Unna und mir nachgewiesen, dass der Sklerose gewisse charakteristische Eigenschaften innewohnen, welche sie von einfachen syphilitischen Infiltraten, Papeln, Knoten u. s. w. auch anatomisch scharf unterscheiden.

1) Die Lehren vom syphilitischen Contagium und ibre thatsächliche Begründung. Wien 1866 bei Braumüller, pag. 353 u. ff. 
Wenn somit der Autor auf der Impfstelle nach drei Wochen Papeln auftreten sah - wie so viele andere Experimentatoren -, so hat dies mit der Natur, der Bedeutung und Prognose der Initialsklerose nicht das Mindeste zu thun und os ist für die letztere irrelevant, ob man mit Zeissl diese Papeln für den Ausdruck der schon vollzogenen Allgemeininfection halten will oder nicht.

Es geht aber aus dem eben Gesagten deutlich hervor, wie sehr die unbedingte Hingabe an eine Theorie, in diesem Falle an die Baerensprung'sche dualistische Lehre mit ihren scharfen, fast tyrannischen Formulirungen, den Blick trübt und ihn dio wichtigsten Untersehiede übersehen macht. Ist man einmal recht fest überzeugt, dass alle an den Uebertragungsstellen auftretenden Veränderungen Symptome der Allgemeininfection seien, dann geräth man auch leicht dahin, diese Veränderungen mit einander zusammenzuwerfen und ihre anatomischen und klinischen Differenzen vollkommen $\mathrm{zu}$ ignoriren.

Fragen wir uns nun aber, worin denn eigentlich die Beweiskraft des obigen Experiments gelegen sein soll, wenn weder der Erfolg der Probeimpfung an sich, noch die Beschaffenheit dor localen Infiltrate an sich einen solchen Beweis berzustellen vermochten? Offenbar darin, dass das Nichtanschlagen der Probeimpfung, welches Z. für den Ausdruck der völligen Sättigung des Organismus mit Syphilis ansieht, zu einer Zeit stattfand, wo die Leistendrüsen noch gar nicht geschwollen und von sonstigen allgemeinen Symptomen gar nichts sichtbar war ${ }^{1}$ ). Damit wäre nun aber im äussersten Falle nur der Nachweis geliefert, dass die Durchsenchung des Organismns zur Zeit der Probeimpfung vollzogen war; ob dies aber schon früher und ob es zur Zeit des Auftretens der Papeln der Fall gewesen sei, wie Zeissl ausdrücklicb folgert, lernen wir daraus keineswegs, um so weniger, als nicht einmal angegeben wird, ob die Probeimpfungen vor oder nach dem Auftreten der Papeln stattgefunden haben. Es wird nur gesagt, sie wären vor dem Erscheinen der Leistenbubonen vorgenommen worden. War aber der

1) Vom weiteren Verlaufe des Falles und ob iberhanpt Roseola, Angina u. s. w. später anfgetreten seien, erfahren wir merkwürdiger Weise überhaupt nichts. 
Organismus schon vor dem Auftreten der letzteren durchseucht, so fällt damit die ganze Theorie von dem Fortschreiten des Contagiums durch die Lymphwege ins Blut, von seiner Deponirung in den Lymphdrüsen während dieses Marsches und von dem anfallsweisen Herausstürmen des Giftes aus seinen Depôts bei den jeweiligen Syphilis-Eruptionen ${ }^{\mathrm{i}}$ ).

Es bliebe somit dem Berichterstatter über das obige Experiment logischer Weise nichts übrig als zu sagen: Das Contagium hat einer gewissen Zeit (Incubation) bedurft, bis es seine giftigen Wirkungen entfalten konnte. Diese Zeit hat es irgendwo zubringen müssen. In den Inguinaldrüsen oder gar jenseits derselben kann dies nicht gewesen sein, weil ja angeblich durch die Probeimpfung sehon vor der Drüsensehwellung die Durchseuchung constatirt worden war. Somit mïsste die Stelle der Ueberimpfung selbst und ihre Umgebung als Sitz der Weiterentwicklung des Contagiums angesehen werden und dies hiesse nichts Anderes als: Die sich dort bildende Affection ist eine Localerscheinung und deren Auftreten weist wohl auf die Wahrscheinlichkeit hin, dass das Contagium auch weiter resorbirt werden könne oder schon resorbirt worden sei - aber diese Sklerose ist nicht etwas mit der Localität der Ueberimpfung nur zufällig Zusammenhängendes, ein Syphilis-Symptom wie andere mehr, sondern es ist die Brütstäite des Contagiums.

Ein Dualist darf aber dies nicht zugeben, weil die Sklerose schon einmal unbedingt als Allgemeinsymptom proclamirt ist.

Er wird sich also damit bescheiden müssen, von einer Bestimmung der syphilographischen Länge und Breite ganz abzusehen und einfach zu sagen: Das Gift kommt - auf welchem Wege immer - in die Blutmasse und entfaltet dort seine giftigen Eigenschaften. Dieser möglichst unverfänglichen - weil nichtssagenden - Theorie steht nur freilich die eine Schwierigkeit entgegen, zu erklären, warum die Initialsklerosen - diese eigenthümlichen Gebilde - gerade an den Uebertragungsstellen des Contagiums auftreten, an Stellen, welche ja mit der eigentlichen Präparation des Giftes gar nichts zu thun batten? Mit dem leeren Schlagworte vom Locus minoris resistentiae $\mathbf{u}$. dgl. trösten sich ja hent zu

1) Zeissl ist in der That kein Anbänger dieser Theorie. 
Tage nur mehr wenige Syphilidologen, deren wissenschaftliche Zähigkeit in der That über jedes Schwanken erhaben ist.

$\mathrm{Zu}$ diesen zählt nun freilich unser Autor in diesem Falle nicht; or verschmäht es dies Mal nicht, rechtzeitig Concessionen zu machen, eingedenk des Ricord'schen Satzes: „L'homme absurde est celui, qui ne change jamais". Und so stellt er denn zum Schlnsse seines Artikels eine neue Hypothese auf, welche besagt, dass nur ein kleinerer Theil der krankmachenden Mikroorganismen - man sieht, dass der Autor vor Hypothesen überhaupt nicht zurücksehreckt - von der Impfstelle aus resorbirt werde und in den Krejslauf gelange, der grössere Theil aber an der Impfstelle liegen bleibe. "Selbstrerständlich werden nun die meisten Mikroorganismen sich dort entwickeln, wo von Anfang an die meisten gewesen sind, das ist an der Impfstelle, und werden an dieser auch zuerst ihr Krankheitsprodukt, das Zeichen ihrer Anwesenheit, zeitigen; es ist dies die syphilitische Initialsklerose oder die initiale Papel. Die in den Blutkreislauf gelangten Mikroorganismen werden sich unter den gleichen günstigen $\mathrm{Be-}$ dingungen ebenfalls vermehren. Um unserem Auge wahrnehmbare Erscheinungen hervorzurufen, braucht es aber wahrscheinlich einer bestimmten Quantität solcher Mikroorganismen und bis diese Menge durch Vermehrung der in Folge der Einimpfung in den Kreislauf gelangten Organismen sich entwickelt hat, verstreicht ein längerer Zeitraum, als wie die Mikroorganismen brauchten, um die initiale Geschwulst an der Impfstelle zu erzeugen."

Ich erlaube mir nur noch die eine Frage: Verlobnt es sich der Mühe, wenn man einmal zu einer solchen Opportunitätstheorie gelangt ist, die alte dualistische Lehre von der Allgemeinnatur der Initialsklerose aufrecht zu erhalten? Steckt in der neuen Hypothese nicht schon die Ueberzengung, dass es sich bei dem ganzen Vorgang der sypliilitischen Durchseuchung nur um ein schrittweises Vorwärtsschreiten des Contagiums von der Impfstelle aus handle? Und wäre es nicht einfacher und natürlicher zuzugeben, dass die Fertigmachung des Contagiums auch für den ganzen Organismus an der Impfstelle selbst erfolgen könne, und dass dieses dann anzunehmen sei, wemn sich an dieser Stelle 
die allmälige Entwickelung der Initialsklerose mit den ihr eigenthümlichen von Unna und mir beschriebenen Gefässwandveränderungen vollzieht? Dass es auch Fälle von Syphilis-Uebertragung gibt, wo weder eine solehe Sklerosirung, noch überhaupt ein sichtbares Symptom an der Impfstelle auftritt, haben ja gerade die Gegner der Dualitätslebre am wenigsten geläugnet.

Ich schliesse hiermit diese Bemerkungen, welche niederzuschreiben ich mich verpflichtet hielt. Denn der Kampf zwischen neuen Vorstellungen und alten, in das Blut übergegangenen Theorien muss - so schwierig und unerquicklich er ist - ausgefochten, und es darf nicht ohne Gegenwebr zugelassen werden, wenn dieser Kampf von einer in der Gelehrtenrepublik respectirten Seite her mit Waffen geführt wird, welche im Gebiete der Naturwissenschaft heutzutage nicht meinr als regulär angesehen werden. 